\title{
Effects of high external electric fields on protein conformation
}

\author{
Pier Paolo Pompa ${ }^{* a, b}$, Alessandro Bramanti ${ }^{\mathrm{a} \dagger}$, Giuseppe Maruccio ${ }^{\mathrm{a}}$, Loretta Laureana del Mercato ${ }^{\mathrm{a}, \mathrm{b}}$, \\ Rocco Chiuri ${ }^{\mathrm{a}}$, Roberto Cingolani ${ }^{\mathrm{a}}$, and Ross Rinaldi ${ }^{\mathrm{a}}$

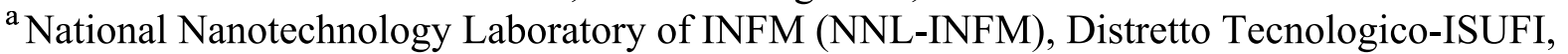 \\ Università di Lecce, Via Arnesano, 73100 Lecce, ITALY \\ ${ }^{\mathrm{b}}$ ISUFI, Istituto Superiore Universitario per la Formazione Interdisciplinare, Via Arnesano, 73100 \\ Lecce, ITALY
}

\begin{abstract}
Resistance of biomolecules to high electric fields is a main concern for nanobioelectronics/nanobiosensing applications, and it is also a relevant issue from a fundamental perspective, to understand the dielectric properties and structural dynamics of proteins. In nanoscale devices, biomolecules may experience electric fields as high as $10^{7} \mathrm{~V} / \mathrm{m}$ in order to elicit charge transport/transfer. Understanding the effects of such fields on their structural integrity is thus crucial to assess the reliability of biomolecular devices. In this study, we show experimental evidence for the retention of nativelike fold pattern by proteins embedded in high electric fields. We have tested the metalloprotein azurin, deposited onto $\mathrm{SiO}_{2}$ substrates in air with proper electrode configuration, by applying high static electric fields (up to $10^{6}-10^{7} \mathrm{~V} / \mathrm{m}$ ). The effects on the conformational properties of protein molecules have been determined by means of intrinsic fluorescence measurements. Experimental results indicate that no significant field-induced conformational alteration occurs. This behavior is also discussed and supported by theoretical predictions of the intrinsic intra-protein electric fields. As the general features of such inner fields are not peculiar of azurin, the conclusions presented here should have general validity.
\end{abstract}

Key words: Protein Conformation, Electric Fields, Fluorescence Spectroscopy, Biomolecular Electronics.

\section{INTRODUCTION}

Molecular bioelectronics is a rapidly growing field at the junction of biochemistry, physics and surface science, which integrates biomaterials with electronic transducers ${ }^{1,2}$. The molecular building blocks of life - as proteins and nucleic acids - are examples of biomaterials that possess unique properties determined by their size, folding and patterns at the nanoscale. Thanks to their properties, proteins and enzymes are extremely interesting for nanobiotechnology and nanoelectronics because their great potential in controlling highly specific reactions can be optimized towards different technological targets. Integrating biological building blocks into synthetic materials and devices allows combining natural biological functions, such as binding, catalysis, recognition, self assembly and electron transfer (ET), with the processing power of modern microelectronics for the realization of biosensors, devices for driving biotransformations, and eventually biocomputers ${ }^{3,4}$. The possible applications involve drug and gene delivery, biocompatible nanostructured materials for implantation, artificial photosynthesis for clean energy, and nanoscale sensory systems, with considerable advantages in several fields such as medicine, environmental diagnostics and food and processing industries.

In this work we have focused the attention on the function of natural electron-transfer proteins. The ET is a key phenomenon since it can be exploited in many reactions of biotechnological interest. The research on protein film assemblies on solid substrates is fundamental for those bioelectronic applications which aim to substitute inorganic semiconductors materials with biomolecular conductive materials in electronic devices ${ }^{5-8}$.

In particular, redox metalloproteins have gained considerable interest for their electric properties ${ }^{9,10}$. Indeed, their inherent ET capability (thanks to the presence of a redox center) is very efficient. The peculiarity of ET processes mediated by metalloproteins is that they occur over long distances and in a very fast, directional way. Metalloproteins are, in general, part of ET chains where the conduction through the biomolecule occurs at the single electron scale ${ }^{11,12}$. Moreover, their transport properties can be regulated by controlling the redox activity, so that applications as electronic

\footnotetext{
*email:piero.pompa@unile.it; phone 0039-0832-298382; fax 0039-0832-298238; www.nnl.it

†presently with STMicroelectronics
} 
switches are possible. All these characteristics make them good candidates for incorporation in hybrid submicrometersized electronic components ${ }^{13-17}$.

The blue copper protein Pseudomonas aeruginosa azurin (Az) is one of the best-characterized redox metalloproteins. This small molecule, (MW=14.6 kD) consisting of 128 amino acid residues, plays an important role as electron carrier in the respiratory chain of denitrifying bacteria, where its role is to transport electrons between cytochrome c551 and nitrite reductase. The active site is located in a hydrophobic core approximately $7 \AA$ far from protein surface ${ }^{18}$ and consists of a single copper ion (having five amino acid ligands arranged in a trigonal bipyramidal geometry) switching between $\mathrm{Cu}$ (I) and $\mathrm{Cu}$ (II) oxidation states during the ET process. The redox-mediated electron transfer is favoured by the minimal structural differences of the metalloprotein in the two states of the copper ion. Thanks to such characteristics, azurin has recently been employed with success as the active element of rectifying (diode-like) and modulating (transistor-like) biomolecular devices ${ }^{19-22}$.

In order to develop protein-based devices, the structural stability of biological macromolecules in solid state and ambient conditions has to be studied. In particular, investigations about resistance to high electric fields as well as ageing effects are of fundamental and applicative interest (e.g. biomolecular electronic devices), because the high electric fields applied to nanometric devices are frequently indicated as potential sources of rapid molecular degradation and biological macromolecules may not be structurally stable in ambient conditions (in air). A schematic representation of a typical configuration for nanoscaled biomolecular devices is shown in Fig. 1. As a general rule, the inter-electrode separation is in the range of 10-100 nanometers and so the application of bias potential of few volts results in the genearation of electric fields up to $10^{7} \mathrm{~V} / \mathrm{m}$. Understanding the effects of such fields on the structural integrity of biomolecules is thus crucial to assess the reliability of hybrid devices.

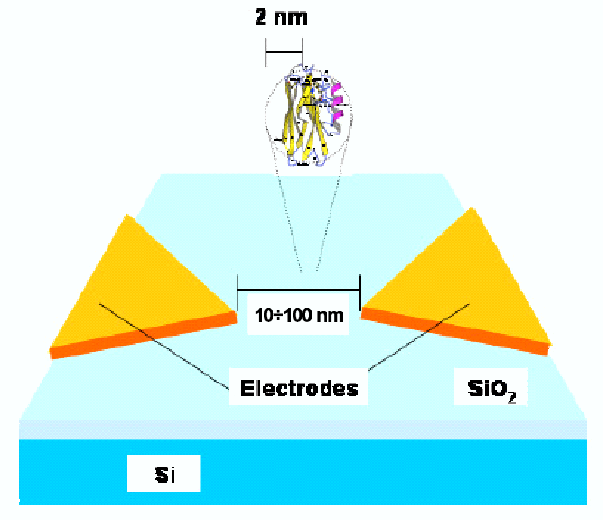

Figure 1. Graphic representation showing typical nanoscaled biomolecular devices in which a protein is immobilized between two metallic tips. The distance between the electrodes is evidenced.

In this work, we have studied the conformational features of solid-state films of Az protein from $P$. aeruginosa in order to verify the suitability of this molecule for the realization of protein-based devices. In particular, to measure the resistence of proteins to high external electric fields, we investigated the possible changes of the protein conformational properties under high static electric fields (up to $10^{6}-10^{7} \mathrm{~V} / \mathrm{m}$ ). We tested the apo-protein form (without $\mathrm{Cu}^{2+}$ ) of azurin deposited onto $\mathrm{SiO}_{2}$ substrates in air with proper electrode configuration. In addition, we studied ageing effects onto the same samples at ambient conditions, in absence of electric field, over several weeks. All measurements were carried out by means of highly sensitive fluorescence spectroscopy experiments.

The intrinsic fluorescence spectrum of $P$. aeruginosa azurin, a sensitive probe of the protein conformation ${ }^{23}$, is due to a single tryptophan residue (Trp48) ${ }^{24}$. Due to the highly hydrophobic microenvironment surrounding Trp48, the emission peak is centered at a remarkably short wavelength $\left(\lambda_{\max } \approx 306-308 \mathrm{~nm}\right)^{24-26}$; this is consistent, on the other hand, with previous crystal structure studies ${ }^{26-29}$. In order to assess the immobilization of azurin protein after deposition onto the interdigitated electrodes, tapping mode atomic force microscopy (TM-AFM) was used ${ }^{30,31}$. 
Experimental results indicate that no significant field-induced conformational alteration occurs. Such results are also discussed and supported by theoretical predictions of the inner protein fields. Moreover, no significant structural changes due to ageing effects occur in the metalloprotein maintained at ambient conditions.

\section{EXPERIMENTAL}

\subsection{Electrodes fabrication}

Figure 2 displays the optical micrograph of the interdigitated electrodes used in the fluorescence experiments. These devices were fabricated on thermally oxidized silicon wafers, using standard photolithographic techniques. The structure, defined in AZ5214 resist, consisted of 500 interdigited gold electrodes with a line-space period of $2 \mu \mathrm{m}$, covering an active area of $1 \times 1 \mathrm{~mm}^{2}$. After exposure, samples were developed in AZ726MIF. The metal electrodes were obtained by e-beam metal evaporation $(\mathrm{Cr} / \mathrm{Au})$ followed by lift-off in acetone bath for more than 10 min at moderate temperature $\left(35-40^{\circ} \mathrm{C}\right)$.

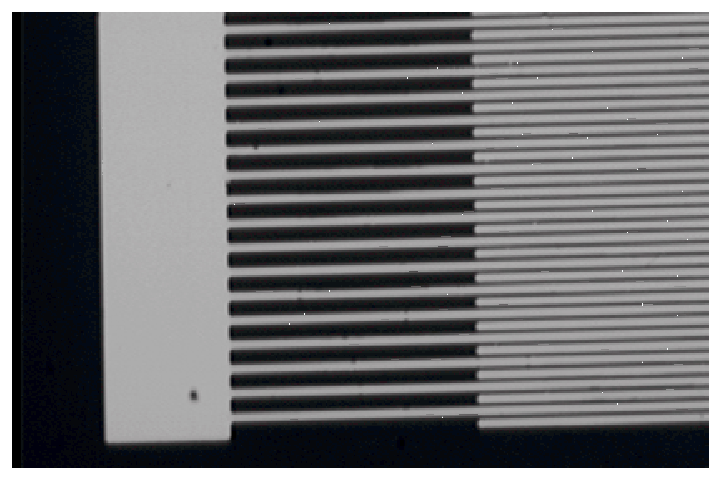

Figure 2. Photomicrograph of the interdigitated electrodes used in experiments to check the integrity of the protein films subjected to high electric fields.

\subsection{FEM analysis}

The electrodes geometry was accurately designed both to allow the application of high electric fields to protein molecules and to detect the very weak fluorescence signal over a large area. The entity and spatial distribution of the electric field generated in the electrodes, where the protein was immobilized, were assessed by a finite element electrostatic simulation of the electrodes. To this purpose, a 3D geometry was defined in order to evaluate the bending close to the upper border of the electrodes. Fixed potential values were assigned to the electrodes, while a weak Neumann boundary condition (i.e., null tangential field) was imposed on the other bounderies. The inter-electrode material was chosen to be vacuum.

\subsection{Protein immobilization and AFM characterization}

Azurin samples from Pseudomonas aeruginosa were kindly supplied by Prof. G.W. Canters (Leiden Institute of Chemistry, Leiden, NE). The protein was purified by chromatography using carboxymethyl (CM) Sepharose Fast Flow (FF) and diethylaminoethyl (DEAE) Sepharose. The product was characterized by sodium dodecylsulfate poly(acrylamide) gel electrophoresis (SDS-PAGE), and stored at - $20^{\circ} \mathrm{C}$ in $20 \mathrm{mM}$ 4-(2-hydroxyethyl)-1piperazineethanesulfonic acid (HEPES) $\mathrm{pH} 7$ buffer.

The protein immobilization was obtained by cast deposition of a $20 \mu \mathrm{ldrop}$ of the protein solution $(1.0 \mathrm{mg} / \mathrm{ml}$ in $50 \mathrm{mM}$ $\mathrm{NH}_{4} \mathrm{Ac}$ buffer, $\mathrm{pH} 4.6$ ) onto the interdigited electrodes structures; the incubation time was 15 min (at room temperature). After incubation, the buffer solution was removed, and the samples were accurately dried by high purity nitrogen flow. The presence of azurin proteins adsorbed on substrates was demonstrated by in situ AFM experiments. TM-AFM images were performed with a BioScope microscope (Digital Instruments) equipped with a $90 \mu \mathrm{m}$ scanner operating in tapping mode at a resonance frequency of $\sim 320 \mathrm{kHz}$. Silicon nitride probes were used. All protein imaging was performed in air at room temperature. Before engaging, scan size and offsets were set to zero to minimise sample deformation and 
contamination of the tip. In each measurement, the set point was adjusted before scanning, to minimise the force between the tip and the sample. The typical scan rate was $0.5 \mathrm{~Hz}$.

\subsection{Fluorescence spectroscopy}

Fluorescence spectra were recorded in photon counting mode, by using a $450 \mathrm{~W}$ Xenon lamp as the source of excitation and double monochromators both in excitation and emission. The excitation wavelength was $280 \mathrm{~nm}$ ( $2 \mathrm{~nm}$ bandwidth). For measurements in solution, the emitted light was observed at right angles to the excitation radiation. Photoluminescence spectra of control samples, without protein, were recorded and subtracted from the experimental samples to correct for background interference. To monitor azurin luminescence from protein in the solid state, the exciting radiation was focused on the protein film with $30^{\circ}$ angle of incidence, while the front surface emission was collected at $90^{\circ}$ from the excitation. The intensity losses were negligible or less than $5 \%$ depending on the experimental conditions, thus excluding appreciable Trp photodegradation during acquisition. All the experiments were performed at room temperature $\left(20^{\circ} \mathrm{C}\right)$, atmospheric pressure, and $54 \%$ of humidity.

\subsection{Evaluation of intraprotein electric field: MEP analysis}

The internal electric field of the protein was estimated from the spatial gradient of the molecular electrostatic potential (MEP) calculated for the structure of oxidized P. aeruginosa azurin in solution at pH 5.5 (molecule A in the PDB file 4azu. http://pdb.gmd.de). The linearized Poisson equation was solved by means of the University of Houston Brownian Dynamics (UHBD) software ${ }^{32}$, assuming as source the charge distribution on the residues ${ }^{19}$ determined by X-ray structural analysis. A $1 \AA$-stepped computational grid was the frame, both for the MEP and field calculation. The adopted model provides a first order estimate of the inner electric field. The comparison with applied fields, though qualitative, is sufficient for drawing reliable conclusions on resilience of the molecule.

\section{RESULTS}

\subsection{Simulation of spatial distribution of the electric field in the devices}

We have studied the influence of strong electric fields on the protein conformation by means of interdigitated electrodes. The electric field distribution in the realized structure is displayed in Fig. 3. The figure displays the color map of the field intensity obtained by FEM analysis, on a cross-section of the interdigitaded electrodes (growing values from blue to red), reported in Fig. 2.

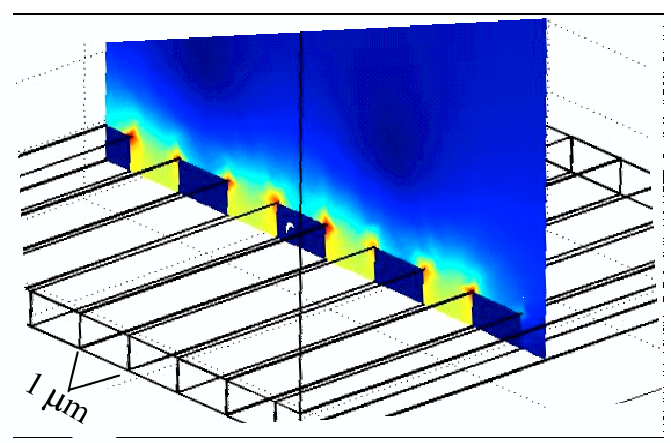

Figure 3. Map of the applied electric field obtained by the electromagnetic field simulation using finite element methods. The colour scale ranges from blue to red following the visible spectrum, with the red region corresponding to the highest field. Between the electrodes, a nearly uniform field region may be observed, except for the hotspots on the corners (red).

As shown, the field decays rapidly immediately outside the inter-electrode region (where the field is predictably nearly uniform, except for the hot-spots on the corners, due to accumulated charge). The inter-electrode separation (1 micron) implies the attainment of fields up to $10^{7} \mathrm{~V} / \mathrm{m}$ by applying tens of volts. FEM simulations demonstrated that the electric field between pair of adjacent electrodes is practically uniform. 


\subsection{Protein immobilization and AFM measurements}

As described above, protein molecules were immobilized onto a $\mathrm{SiO}_{2}$ substrate, on which gold interdigitated electrodes were present. Obviously, this kind of immobilization results in protein adsorption both between electrodes, onto the $\mathrm{SiO}_{2}$ substrate, and on top of the electrodes themselves. Therefore, in order to assess the deposition of azurin molecules between the micrometer-sized electrodes, we used the Tapping Mode Atomic Force Microscopy (TM-AFM). In tapping mode, shear forces are greatly reduced thanks to the intermittent contact between tip and sample: the cantilever oscillates vertically by a piezoelectric crystal, just barely "tapping" the sample, and the reduction in oscillation amplitude is used to identify and measure surface features ${ }^{30}$. This powerful technique allows high resolution topographic imaging of sample surfaces that are easily damaged, or difficult to image by other AFM techniques ${ }^{31}$.

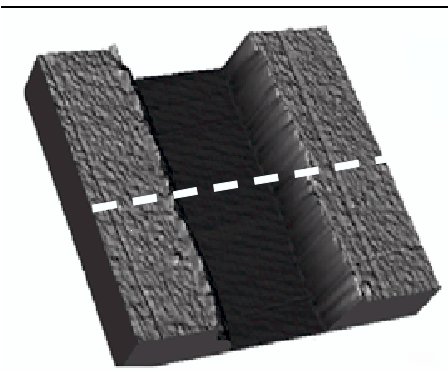

(a)
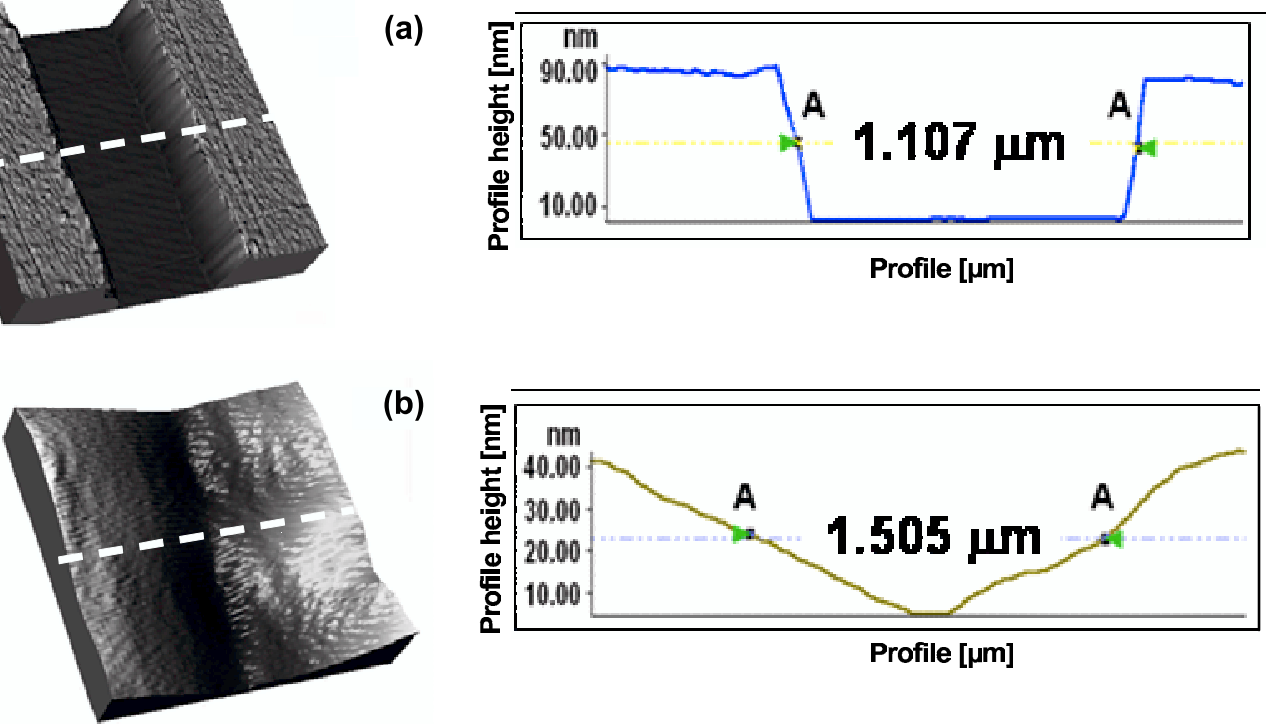

Figure 4. AFM images and profiles of electrodes before (a) and after (b) azurin deposition. The measurements were carried out operating in tapping mode and ambient conditions.

We measured the surface topography of the electrodes before the azurin immobilization, assessing both the height and the separation of the electrodes by cross-section analysis. The measured values were about $90 \mathrm{~nm}$ and $1.107 \mu \mathrm{m}$, respectively (see figure $4 \mathrm{a}$ ). Then, we performed the same measurements after cast deposition of azurin. The crossprofile analysis displayed an apparent electrode height reduction (see figure $4 \mathrm{~b}$ ). This result is explained by a preferential adsorption of azurin in the inter-electrode region. However, an important point is that the external field atop the electrode surface (which is $50-60 \%$ of the peak value) extends, with a very good uniformity, for more than $100 \mathrm{~nm}$ from the metal surface (according to the FEM analysis): this means that we have the same electric field for more than 25 protein layers $(1$ protein $=4 \mathrm{~nm})$ onto the electrodes, i.e., remarkably more than the thickness reached in our experiments. Therefore, the photoluminescence signals collected in our experiments includes contributions both from the protein molecules between the electrodes (which experience the full intensity of the applied electric field) and from molecules atop the electrode surfaces.

\subsection{Emission spectra of azurin under high external electric fields}

We utilized the potential of fluorescence spectroscopy for probing the conformational and structural states that azurin molecules undergo under external electric fields. All proteins contain natural chromophores absorbing in the near ultraviolet, notably the aromatic residues phenylalanine, tyrosine, and tryptophan. In particular, intrinsic fluorescence of $P$. aeruginosa azurin is due to a single tryptophan residue (Trp48) which exhibits a typical "blue" emission $\left(\lambda_{\max } \sim 306 \div 308 \mathrm{~nm}\right)$ when excited in the ultraviolet spectral range $\mathrm{e}^{25}$. This emission has been extensively investigated and 
was demonstrated to be identical for the native state of wild-type azurin, apo-azurin (i.e., without $\mathrm{Cu}^{2+}$ ) and other metal derivatives (such as $\mathrm{Zn}^{2+}$ ) ${ }^{25-26}$. However, the different derivatives are charachterized by large variations in the fluorescence quantum yield ${ }^{33}$ because of a strong quenching mechanism by the metal ions. For this reason, in this work we used the apo form.

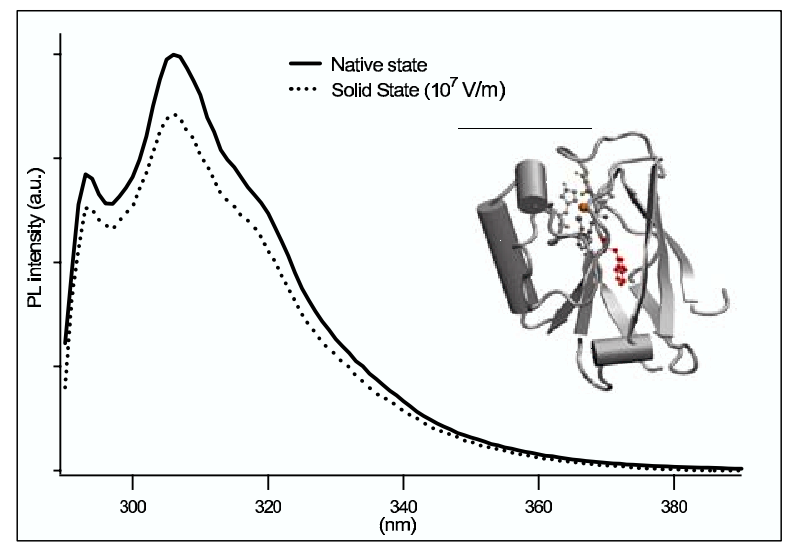

Figure 5. Intrinsic fluorescence spectra of apo-azurin in the native state and in the film environment with an applied external electric field (the fluorescence intensities are not in scale). It is evident that the protein preserves its characteristic fluorescence spectrum even after exposure to $10^{7} \mathrm{~V} / \mathrm{m}$ electric field. Inset: structure of azurin with focus on the relative arrangement of Trp48 and the electron-transfer active site. The residue Trp48, which is responsible for luminescence, is shown in red (in ball\&stick rendering). The active site is shown in gray (the large orange ball represents the $\mathrm{Cu}$ atom). The rest of the protein is shown with the conventional features of the secondary structure, $\alpha$-helices and $\beta$-sheets, backbone.

The intrinsic fluorescence in azurin is very sensitive to small perturbations of the protein folding ${ }^{23}$, so that any significant conformational variation can be efficiently detected by this spectroscopic analysis. We compared the emission spectrum of apo-azurin protein in native state (solid line in figure 5) with the emission spectrum of the solid-state protein under a $10^{7} \mathrm{~V} / \mathrm{m}$ external electric field (dashed line in figure 5): as shown, there is no significant difference in the two observed line-shapes. Thereby, we have inferred that such electric fields do not affect the overall fold pattern, indicating that the Trp residue remains embedded in the same hydrophobic environment.

Moreover, we probed the sensitivity of the photoluminescence line-shape as a function of excitation wavelength. Azurin in the folded state shows the same emission spectrum independently from the excitation wavelength, whereas spectral line-shapes of unfolded azurin detected at different $\lambda_{\text {exc }}$ can be very different. Photoluminescence experiments on protein films (not shown) at various excitation wavelengths did not reveal any spectral variation, thus confirming the retention of the native-like conformation of azurin in our samples.

The retention of the native-like conformation in Az solid state films is particularly interesting because it reveals the absence of relevant perturbations of the microenvironment surrounding the Trp48 (inset figure 5). This is a significant result also for the electrical properties of azurin, since such residue is thought to play an important role in the long-range ET processes through the molecule ${ }^{25,34}$. This result is important also because it was obtained with the apo derivative, which is characterized by a lower structural stability with respect to the wild-type copper protein ${ }^{23}$. Hence, the preservation of the native-like conformation seems to indicate the lack of gross molecular rearrangements also under extreme experimental conditions, such as those normally achieved in biomolecular electronic devices.

\subsection{MEP analysis}

The calculation described in Section 2.5 yielded a $2.45 \times 10^{9} \mathrm{~V} / \mathrm{m}$ average electric field inside the proteins. Local intensity maxima up to one order of magnitude higher were found, though; see for example the 2D space profiles of field intensity reported in Figure 6, showing peaks as high as $17 \times 10^{9} \mathrm{~V} / \mathrm{m}$. 

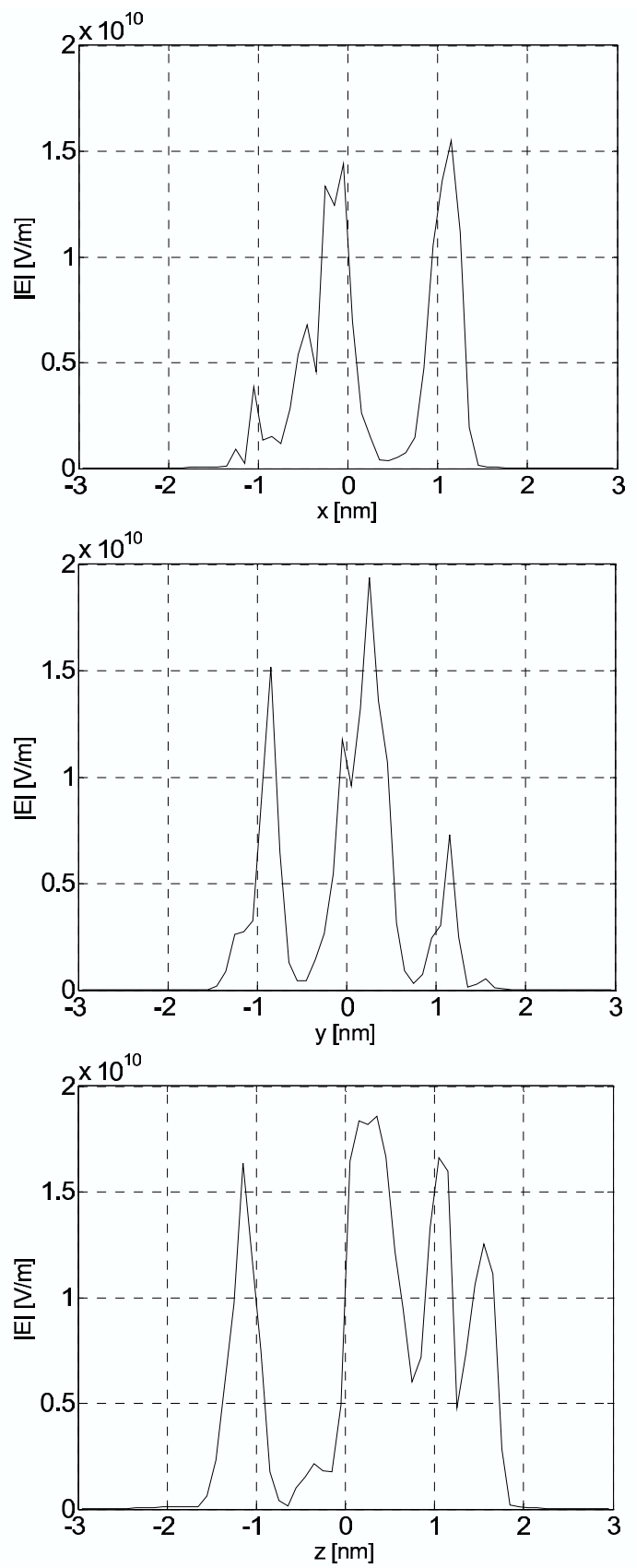

Figure 6. Profiles of the inner electric field in azurin as computed by solving the Poisson equation. The $\mathrm{x}, \mathrm{y}$ and $\mathrm{z}$ axes are respectively represented.

Such inner fields are by orders of magnitude more intensive than those externally applied in our experiments $\left(10^{6}-10^{7}\right.$ $\mathrm{V} / \mathrm{m}$ ), which justifies the lack of perturbation in the fold pattern.

Also, it should be pointed out that our estimates are in quantitative agreement with other recent results. The existence around the protein of a first hydration shel1 135 denser than bulk water was explained as "electrostriction" 36,37 , exerted on the water itself by the surface field of biomolecules; the latter is just estimated in the order of $10^{9} \mathrm{~V} / \mathrm{m}$. Xu et al.$^{38}$ have 
performed molecular dynamics studies on trypsin inhibitor, concluding that only fields above $10^{9} \mathrm{~V} / \mathrm{m}$ can induce structure (or temperature) alteration on the protein, while a $10^{8} \mathrm{~V} / \mathrm{m}$ intensity is within thermal fluctuations. Furthermore, hybrid quantum chemical-molecular dynamics indicate average fields between $5 \times 10^{8}$ and $8.5 \times 10^{9} \mathrm{~V} / \mathrm{m}^{39}$. The univocal conclusion is that azurin is able to retain its conformational state under quite intensive external electric fields, by virtue of even stronger internal fields. Remarkably, however, this is the first experimental evidence in this sense.

\subsection{Ageing of solid state protein films}

We also studied the behavior of azurin in air, at ambient conditions $\left(20^{\circ} \mathrm{C}\right.$, atmospheric pressure, $54 \%$ of humidity). The ageing of protein films was monitored over periods longer then 600 hours, with zero external bias, by intrinsic fluorescence spectroscopy. The figure 7 displays the spectral parameters of apo-azurin versus time, characterizing the conformational state of the protein, i.e. the emission maximum $\left(\lambda_{\max }\right)$ and full width at half maximum $(\mathrm{w})$. These measurements have all been performed as function of time after the drying procedure of the biomolecular films.

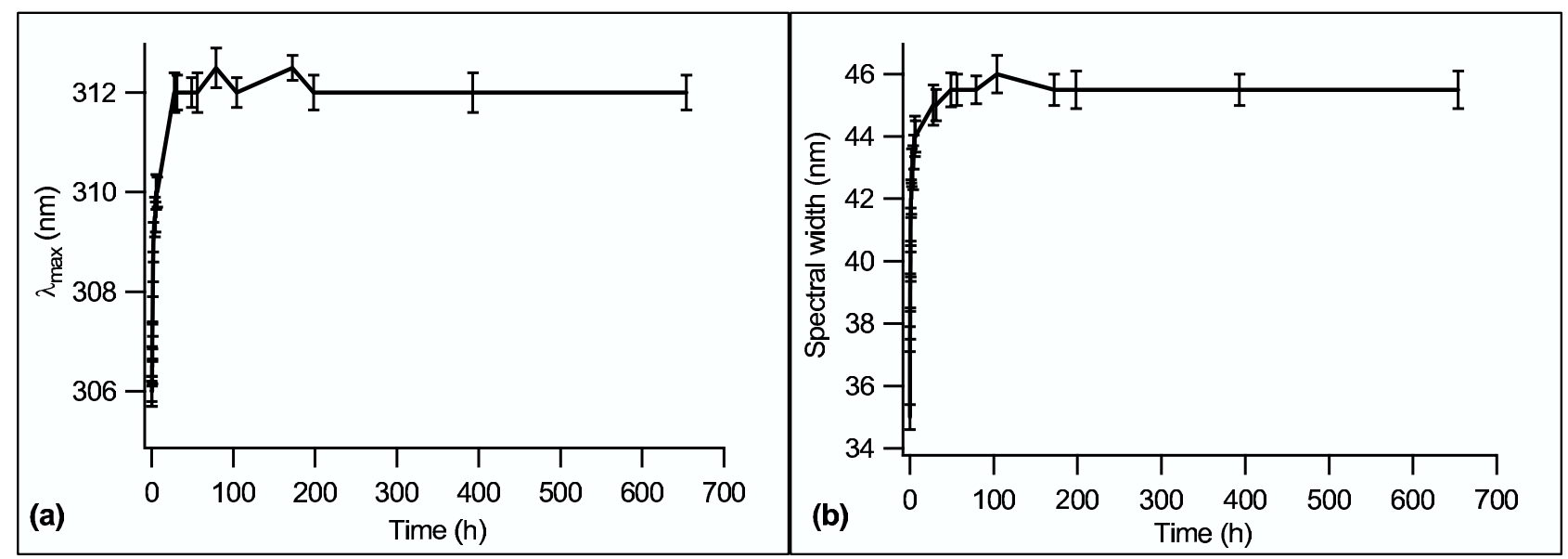

Figure 7. Ageing of apo-azurin at ambient conditions $\left(20^{\circ} \mathrm{C}\right.$, atmospheric pressure, $54 \%$ of humidity), over more than 600 hours, as probed by intrinsic fluorescence experiments. a: emission maximum $\left(\lambda_{\max }\right)$; b: spectral width. The experimental results shown in the figures are the mean values of the data from three independent measurements, and the error bars indicate the standard deviations. After a weak few-hour rearrangement, azurin reaches a stable configuration.

In the first hours we observed a weak modification of the azurin PL, with a slight red-shift which bringing $\lambda_{\max }$ to a maximum of $311 \div 312 \mathrm{~nm}$ (fig. 7a). However, such rearrangement is of modest entity, testifying that the overall fold pattern is not severely perturbed. Interestingly, no other significant conformational changes are observed after the first 100 hours, for up to weeks under non-physiological conditions. Analogous considerations apply to the spectral width (w), which reaches a maximum value of $45 \mathrm{~nm}$ even at the end of the investigated period (Fig. 7b), at variance with the usual values for unfolded azurin $(\mathrm{w} \sim 62 \mathrm{~nm}$ ). It seems that proteins at ambient conditions (room temperature, 50 $\div 60 \%$ relative humidity) reach a stable state, which may be attributed to the capability of azurin of retaining its hydration shell. It is worth noting that upon rehydration at the end of the analyzed period, the typical PL spectrum of native azurin in solution is recovered, indicating the minor scale of the conformational rearrangement observed in the solid state. To our knowledge, this is the first experimental evidence of such effects.

\section{CONCLUSIONS}

We have reported results about resilience of proteins both to the application of high external electric fields and to the prolonged exposition to ambient conditions (in air). The first experiments revealed that proteins in the solid state are capable of maintaining the tightly bound hydration shells and a native-like conformation, even after solvent removal and 
application of fields intensities in the range of $10^{6}-10^{7} \mathrm{~V} / \mathrm{m}$, indicating that no significant field-induced conformational alteration occurs. According to the MEP analysis, such effects can be ascribed to the huge intensity of the inner electric fields $\left(\sim 10^{9} \mathrm{~V} / \mathrm{m}\right)$ present in the proteins. These results give interesting information about structural properties of the investigated protein, confirming their possible application in the field of bioelectronics and biosensors. Moreover, this may also have importance for protein crystallization experiments, since recent studies have demonstrated that the application of high external electric fields (in the range of $10^{5} \mathrm{~V} / \mathrm{m}$ ) during the crystallization of protein samples can positively influence the nucleation rate and kinetics, increasing the size of the crystals and improving their crystallographic quality ${ }^{40}$. As the general features of such inner fields are not peculiar of azurin, the conclusions presented here should have general validity. The second set of measurements - the first systematic experiments on solid state biomolecular ageing - are of high relevance because a strong stability of proteins in non-physiological conditions is demonstrated. In conclusion, azurin is found to be much more reliable a material than might have been expected. These results justify using this protein in biomolecular devices and, somehow support this novel branch of electronics.

\section{ACKNOWLEDGEMENTS}

The authors wish to express their gratitude to Professor G.W. Canters (Leiden Institute of Chemistry, Leiden, NE) who supplied the proteins utilized in our experiments, to Dr. F. Calabi (NNL, Lecce) for helpful discussions, Dr. V. Arima for AFM measurements, and E. D'Amone for technical assistance. We are also grateful to F. De Rienzo, S. Corni and R. Di Felice (INFM-S3) for their contributions in MEP analysis studies. This work has been partially supported by the EU (SAMBA Project).

\section{REFERNENCES}

(1) I. Willner and B. Willner, "Biomaterials integrated with electronic elements: en route to bioelectronics", Trends Biotech., 19, 222-230, 2001.

(2) I. Willner, "Biomaterials for sensors, fuel cells, and circuitry", Science, 298, 2407-2408, 2002.

(3) J.S. Marvin and H.W. Hellinga, "Conversion of a maltose receptor into a zinc biosensor by computational design”, Proc. Natl. Acad. Sci. U S A., 98, 4955-4960, 2001.

(4) L.-Q. Gu, S. Cheley, and H. Bayley, "Capture of a single molecule in a nanocavity", Science, 291, 636-640, 2001.

(5) C. Joachim, J.K. Gimzewski, and A. Aviram, "Electronics using hybrid-molecular and mono-molecular devices", Nature, 408, 541-548, 2000.

(6) J.M. Seminario, A.G. Zacarias, and J.M. Tour, "Theoretical study of a molecular resonant tunneling diode", $J$. Am. Chem. Soc, 122, 3015-3020, 2000.

(7) M.A. Reed, J. Chen, A.M. Rawlett, D.W. Price, and J.M. Tour, "Molecular random access memory cell", Appl. Phys. Lett., 78, 3735-3737, 2001.

(8) G. Maruccio, P. Visconti, V. Arima, S. D'Amico, A. Biasco, E. D’Amone, R. Cingolani, R. Rinaldi, S. Masiero, T. Giorni, and G. Gottarelli, "Field effect transistor based on a modified DNA base", Nano Letters, 3, 479-483, 2003.

(9) C. Lowe, "Nanobiotechnology: the fabrication and applications of chemical and biological nanostructures", Curr. Opin. Struct. Biol., 10, 428-434, 2000.

(10) D.M. Adams, L. Brus, C.E.D. Chidsey, S. Creager, C. Creutz, C.R. Kagan, P.V. Kamat, X.M. Lieberman, S. Lindsay, R.A. Marcus, R.M. Metzger, M.E. Michel-Beyerle, J.R. Miller, M.D. Newton, D.R. Rolison, O. Sankey, K.S. Schanze, J. Yardley, and X. Zhu, "Charge transfer on the nanoscale: current status", J. Phys. Chem. B, 107, 6668-6697, 2003.

(11) K. Sigfridsson, M. Ejdeback, M. Sundahl, and O. Hasson, "Electron transfer in ruthenium-modified spinach plastocyanin mutants", Arch. Biochem. Biophys., 351, 197-206, 1998.

(12) W. Haehnel, T. Jansen, K. Gause, R.B. Klösgen, B. Stahl, D. Michl, B. Huvermann, M. Karas, and R.G. Hermann, "Electron transfer from plastocyanin to photosystem I", EMBO J., 13, 1028-1038, 1994.

(13) G. Gilardi, A. Fantuzzi, and S.J. Sadeghi, "Engineering and design in the bioelectrochemistry of metalloproteins", Curr. Opin. Struct. Biol., 11, 491-499, 2001.

(14) D.I. Gittins, D. Bethell, D.J. Schiffrin, and R.J. Nichols, "A nanometre-scale electronic switch consisting of a metal cluster and redox-addressable groups", Nature, 408, 67-69, 2000. 
(15) A.N. Shipway and I.Willner, "Electronically transduced molecular mechanical and information functions on surfaces", Acc. Chem. Res., 34, 421-432, 2001.

(16) C. Loppacher, M. Guggisberg, O. Pfeiffer, E. Meyer, M. Bammerlin, R. Lüthi, R. Schlittler, J.K. Gimzewski, H. Tang, and C. Joachim, "Direct determination of the energy required to operate a single molecule switch", Phys. Rev. Lett., 90, 066107, 2003.

(17) Y. Xiao, F. Patolsky, E. Katz, J.F. Hainfeld, and I. Willner, "Plugging into enzymes: nanowiring of redox enzymes by a gold nanoparticle", Science, 299, 1877-1881, 2003.

(18) P. Facci, D. Alliata, and S. Cannistraro, "Potential-induced resonant tunneling through a redox metalloprotein investigated by electrochemical scanning probe microscopy", Ultramicroscopy, 89, 291-298, 2001.

(19) R. Rinaldi, A. Biasco, G. Maruccio, R. Cingolani, D. Alliata, L. Andolfi, P. Facci, F. De Rienzo, R. Di Felice, and E. Molinari, "Solid-state molecular rectifier based on self-assembled metalloproteins", Adv. Mater., 20, 1453-1457, 2002.

(20) R. Rinaldi, A. Biasco, G. Maruccio, V. Arima, P. Visconti, R. Cingolani, P. Facci, F. De Rienzo, R. Di Felice, E. Molinari, M.P. Verbeet, and G.W. Canters., "Electronic rectification in protein devices", Appl. Phys. Lett., 82, 472-474, 2003.

(21) G. Maruccio, A. Biasco, P. Visconti, A. Bramanti, P. P. Pompa, F. Calabi, R. Cingolani, R. Rinaldi, S. Corni, R. Di Felice, E. Molinari, M.P. Verbeet, and G.W. Canters, "Towards protein field-effect transistors: report and model of a prototype", Adv. Mater., 17, 816-821, 2005.

(22) R. Rinaldi and R. Cingolani, "Electronic nanodevices based on self-assembled metalloproteins", Phys. E., 21, 45-60, 2004.

(23) J. Leckner, N. Bonander, P. Wittung-Stafshede, B.G. Malmstrom, and B.G. Karlsson, "The effect of the metal ion on the folding energetics of azurin: a comparison of the native, zinc and apoprotein", Biochim. Biophys. Acta, 1342, 19-27, 1997.

(24) A. Finazzi-Agrò, G. Rotilio, L. Avigliano, P. Guerrieri, V. Boffi, and B. Mondovì, "Enviroment of copper in Pseudomonas aeruginosa azurin: fluorometric approach", Biochemistry, 9, 2009-2014, 1970.

(25) S.J. Kroes, G.W. Canters, G. Gilardi, A. van Hoek, and A.J. Visser, "Time-Resolved fluorescence study of azurin variants: conformational heterogeneity and tryptophan mobility”, Biophys. J., 75, 2441-2450, 1998.

(26) H. Nar, A. Messerschmidt, R. Huber, M. van de Kamp, and G.W. Canters, "Crystal structure analysis of oxidized Pseudomonas aeruginosa azurin at $\mathrm{pH} 5.5$ and $\mathrm{pH}$ 9.0. A pH-induced conformational transition involves a peptide bond flip", J. Mol. Biol., 221, 765-772, 1991.

(27) E.N. Baker, "Structure of azurin from alicaligenes denitrificans refinement at 1.8 A resolution and comparison of the two crystallographically independent molecules", J. Mol. Biol., 203, 1071-1095, 1988.

(28) H. Nar, A. Messerschmidt, R. Huber, M. van de Kamp, and G.W. Canters, "Crystal structure of Pseudomonas aeruginosa apo-azurin at 1.85 A resolution”, FEBS Lett., 306, 119-124, 1992.

(29) H. Nar, R. Huber, A. Messerschmidt, A.C. Filippou, M. Barth, M. Jaquinod, M. van de Kamp, and G.W. Canters, "Characterization and crystal structure of Zinc azurin, a by-product of heterologous expression in Escherichia coli of Pseudomonas aeruginosa copper azurin”, Eur. J. Biochem., 205, 1123-1129, 1992.

(30) Q. Zhong, D. Innis, K. Kjoller, and V.B. Elings, "Fractured polymer/silica fiber surface studied by tapping mode atomic force microscopy", Surf. Sci., 290, L688-L692, 1993.

(31) C.A. Putman, K.O. van der Werf, B.G. de Grooth, N.F. van Hulst, and J. Greve, "Viscoelasticity of living cells allows high resolution imaging by tapping mode atomic force microscopy”, Biophys. J., 67, 1749-1753, 1994.

(34) S. Larsson, A. Broo, and L. Sjölin, "Connection between structure, electronic spectrum and electron-transfer properties of blue copper proteins", J. Phys. Chem., 99, 4860-4865, 1995.

(35) D.I. Svergun, S. Richard, M.H.J. Koch, Z. Sayers, S. Kuprin, and G. Zaccai, "Protein hydration in solution: experimental observation by x-ray and neutron scattering", Proc. Natl. Acad. Sci. USA, 95, 2267-2272, 1998.

(36) F. Merzel and J.C. Smith, "Is the first hydration shell of lysozyme of higher density than bulk water?", Proc. Natl. Acad. Sci. USA, 99, 5378-5383, 2002.

(37) I. Danielewicz-Ferchmin, E. Banachowicz, and A.R. Ferchmin, "Protein hydration and the huge electrostriction", Biophys. Chem., 106, 147-153, 2003. 
(38) D. Xu, J.C. Phillips, and K. Schulten, "Protein response to external electric fields: relaxation, hysteresis and echo", J. Phys. Chem., 100, 12108-12121, 1996.

(39) P.R. Callis and B.K. Burgess, "Tryptophan fluorescence shifts in proteins from hybrid simulations: an electrostatic approach", J. Phys. Chem. B, 101, 9429-9432, 1997.

(40) C. Charron, C. Didierjean, J.P. Mangeot, and A. Aubry, "The 'Octopus' plate for protein crystallization under an electric field", J. Appl. Cryst., 36, 1482-1483, 2003. 